\title{
The light filament as vector solitary wave
}

\author{
Lubomir M. Kovachev \\ Institute of Electronics, Bulgarian Academy of Sciences, \\ Tzarigradcko shossee 72,1784 Sofia, Bulgarid*
}

(Dated: April 23, 2022)

\begin{abstract}
We present an analytical approach to the theory of nonlinear propagation of femtosecond optical pulses with broad-band spectrum in gases. The vector character of the nonlinear third-order polarization is investigated in details, taking into account the carrier to envelope phase. The corresponding system of vector amplitude equations is written by using left-hand and right-hand circular components of the electrical field. We found that this system nonlinear equations admits $3 D+1$ vector soliton solution with Lorentz shape. The solution presents relatively stable propagation and rotation with $\mathrm{GHz}$ frequency of the vector of the electrical field in plane, orthogonal to the direction of propagation. The evolution of the intensity profile demonstrate weak self-compression and week spherical wave in the first milliseconds of propagation.
\end{abstract}

PACS numbers: 42.65.Sf, 52.38.Hb

\section{INTRODUCTION}

When a femtosecond laser pulse with power above the critical for self-focusing propagates in air, a number of new physical effects have been observed, such as long-range self-channeling 1, 2], coherent and incoherent $\mathrm{GHz}$ and $\mathrm{THz}$ emission 3, 4], asymmetric pulse shaping, super-broad spectra [5, 6], self-compression 8], polarization rotation [7] and others. A remarkable effect is also that some of the light pulses propagate over distances of several kilometers in vertical traces, preserving their spectrum and shapes [2]. In one typical experiment in the near zone up to $1-3 \mathrm{~m}$ from the source, when the pulse's intensity exceeds $I>10^{12} \mathrm{~W} / \mathrm{cm}^{2}$, initial self-focusing and self-compressing starts, which leads to enlarging the $k_{z}$ spectrum to broad band asymmetric one $\Delta k_{z} \approx k_{0}$. The process increases the core intensity up to $10^{14} \mathrm{~W} / \mathrm{cm}^{2}$, where a short non-homogenous plasma column in the nonlinear focus is observed. Usually the standard model describing the propagation in the near zone is a scalar spatio-temporal nonlinear paraxial equation including in addition terms with plasma ionization, higher order Kerr terms, multiphoton ionization and others [9, 10]. The basic model works properly in the near zone because of the fact, that paraxial approximation is valid for pulses with narrow-band spectrum $\triangle k_{z}<<k_{0}$. In the far-away zone plasma generation and higher-order Kerr terms are also included as necessary for the balance between the self-focussing and plasma defocussing and for obtaining long range self-channeling in gases. However, the above explanation of filamentation is difficult to be applied in the far-away zone. As reviewed in [10 13] the plasma density at long distances from the source is to small to prevent self-focusing. There are basically three main characteristics which remain the same at these distances - the broad-band spectrum, coherent $\mathrm{GHz}$ generation and the width of the core, while the intensity in a stable filament drops to a value of $10^{12} \mathrm{~W} / \mathrm{cm}^{2}$.
The higher-order Kerr terms for pulses with intensities of order of $I \sim 10^{12} \mathrm{~W} / \mathrm{cm}^{2}$ are also too small to prevent self-focussing. The experiments, where observation of long-range self-channeling without ionization was realized [11 13], show the needing to change the role of the plasma in the laser filamentation. In addition, there are difficulties with the physical interpretation of the coherent $\mathrm{GHz}$ radiation as a result of plasma generation. The light pulse near the nonlinear focus emits incoherent and non-homogenous plasma, while the coherent $\mathrm{GHz}$ radiation requires homogenous plasma with fixed electron density of the order of $10^{15} \mathrm{~cm}^{-3}$. Only homogenous plasma can generate coherent $\mathrm{GHz}$ emission, but such kind of plasma absent in the process of filamentation. In the experiments the following basic characteristic of the long-range filaments in air are detected:

1. Broad-band spectrum $\left(\Delta k_{z} \sim k_{0}\right)$.

2. Intensity a little above of the value for self-focusing $\left(I \cong I_{\text {self }-f o c} \simeq 10^{12} \mathrm{~W} / \mathrm{cm}^{2}\right)$.

3. Absence of plasma at long distances.

4. Asymmetric relatively stable (Lorentz) spectral and longitudinal forms.

5. Coherent $\mathrm{GHz}$ generation.

Recently in [18] was developed scalar ionization-free non-paraxial nonlinear model which gives the above characteristics of the stable filament. The analytical and the numerical results describes correctly the linear and nonlinear evolution of narrow-band and broad-band laser pulses and in addition it was found, that the equation admits exact Lorentz-type soliton solutions in approximation of neglecting the $\mathrm{GHz}$ oscillation. Nevertheless this theory encountered significant difficulties. The main problems are:

1. Peak instability of the soliton solution under small initial perturbations.

2. The soliton solution is obtained after neglecting the $\mathrm{GHz}$ oscillation.

3. The soliton solution admits one free parameter. 
4. There are problems with the conservation law of the nonlinear operator, when we use the $\mathrm{GHz}$ oscillation.

To solve the above problems we propose in this paper a nonlinear vector model.

\section{NONLINEAR POLARIZATION}

The self-action process broadens the pulse spectrum starting with narrow-band pulse, the stable filament becomes broad-band far way from the source. From other hand, the standard filamentation model based on plasma generation and multi-photon processes includes also nonlinear polarization of the kind

$$
\vec{P}^{n l}=n_{2}\left[\left(\vec{E} \cdot \vec{E}^{*}\right) \vec{E}+\frac{1}{2}(\vec{E} \cdot \vec{E}) \vec{E}^{*}\right],
$$

where $n_{2}$ is the nonlinear refractive index of the isotropic media. The polarization (11) was proposed by Maker and Terhune in 1965 [14]. If the electrical field contains one linear or circular component, the polarization (11) describes only the self-action effect, while in the case of two-component electrical filed $\vec{E}=\left(E_{x}, E_{y}, 0\right)$ additional terms appear, presenting cross-modulation and degenerate four-photon parametric mixing. The self-action process broadens the pulse spectrum - starting from narrowband pulse, the stable filament becomes broad-band far way from the source. Later in [15 17] it is shown, that the evolution of broad-band pulses like filaments can not be described correctly by nonlinear polarization of the kind (11). It is more correct to use the generalized nonlinear operator

$$
\vec{P}^{n l}=n_{2}(\vec{E} \cdot \vec{E}) \vec{E}
$$

which includes additional processes associated with third harmonic generation (THG). The more precise analysis, presented in the paper, demonstrates that the polarization of kind (2) is not applicable to a scalar model, because the corresponding Manley-Rowe (MR) conservation laws are not satisfied. That is why we substitute into the nonlinear operators (1) and (2) two-component electrical vector field at one carrying frequency in the form:

$$
\begin{array}{r}
\vec{E}=\frac{\left(A_{x} \exp \left[i k_{0}\left(z-v_{p h} t\right)\right]+c . c .\right)}{2} \vec{x}+ \\
\frac{\left(A_{y} \exp \left[i k_{0}\left(z-v_{p h} t\right)\right]-c . c .\right)}{2 i} \vec{y}
\end{array}
$$

where $A_{x}=A_{x}(x, y, z, t), A_{y}=A_{y}(x, y, z, t)$ are the amplitude functions, $k_{0}$ is carrying wave number, $\omega_{0}$ is the carrying frequency of the laser source and $v_{p h}$ is the phase velocity. Here we take in mind the fact that the $x$ and $y$ components are orthogonal and complex conjugated. In the case of Maker and Terhune polarization (1) we obtain for $A_{x}$ and $A_{y}$ components the following expressions:

$$
\begin{aligned}
\vec{P}_{x}^{n l}=\frac{3}{8} n_{2}\left[\left(\left|A_{x}\right|^{2}+\right.\right. & \left.\left.\frac{2}{3}\left|A_{y}\right|^{2}\right) A_{x}-\frac{1}{3} A_{x}^{*} A_{y}^{2}\right] \times \\
& \times \exp \left[i k_{0}\left(z-v_{p h} t\right)\right]+c . c . \\
\vec{P}_{y}^{n l}=\frac{3}{8 i} n_{2}\left[\left(\left|A_{y}\right|^{2}+\right.\right. & \left.\left.\frac{2}{3}\left|A_{x}\right|^{2}\right) A_{y}-\frac{1}{3} A_{y}^{*} A_{x}^{2}\right] \times \\
& \times \exp \left[i k_{0}\left(z-v_{p h} t\right)\right]+c . c .
\end{aligned}
$$

The nonrestricted nonlinear polarization (2) generates the following components

$$
\begin{array}{r}
\vec{P}_{x}^{n l}=\tilde{n}_{2}\left[\frac{1}{3}\left(A_{x}^{2}-A_{y}^{2}\right) A_{x} \exp \left[2 i k_{0}\left(z-v_{p h} t\right)\right]\right. \\
\left.+\left(\left|A_{x}\right|^{2}+\frac{2}{3}\left|A_{y}\right|^{2}\right) A_{x}-\frac{1}{3} A_{x}^{*} A_{y}^{2}\right] \exp \left[i k_{0}\left(z-v_{p h} t\right)\right]+c . c . \\
\left.+\left(\left|A_{y}\right|^{2}+\frac{2}{3}\left|A_{x}\right|^{2}\right) A_{y}-\frac{1}{3} A_{y}^{*} A_{x}^{2}\right] \exp \left[i k_{0}\left(z-v_{p h} t\right)\right]+c . c .
\end{array}
$$

where $\tilde{n}_{2}=\frac{3}{8} n_{2}$. Comparing (41) and (5), it is clearly seen that the operator (2) $n_{2}(\vec{E} \cdot \vec{E}) \vec{E}$ generalizes the case of Marker and Terhune's operator (1), but includes also additional terms associated with THG.

The generalized nonlinear polarization (2) is quite simple in the terms of left-hand and right-hand circularly components [19, 20]

$$
\begin{array}{r}
E_{+}(x, y, z, t)=A_{+}(x, y, z, t) \exp \left[i k_{0}\left(z-v_{p h} t\right)\right] \\
E_{-}(x, y, z, t)=A_{-}(x, y, z, t) \exp \left[-i k_{0}\left(z-v_{p h} t\right)\right],
\end{array}
$$

where

$$
\begin{aligned}
& A_{+}=\left(A_{x}+i A_{y}\right) / \sqrt{2} \\
& A_{-}=\left(A_{x}-i A_{y}\right) / \sqrt{2}
\end{aligned}
$$

are the left-hand $A_{+}$and right-hand $A_{-}$circular components of the amplitude fields. Using the technique of calculations, presented in [19], we obtain that the nonlinear polarization (2) generates the following components 


$$
\begin{array}{r}
P_{+}=n_{2}\left(A_{+}^{2} A_{-}\right) \exp \left[i k_{0}\left(z-v_{p h} t\right)\right] \\
P_{-}=n_{2}\left(A_{-}^{2} A_{+}\right) \exp \left[-i k_{0}\left(z-v_{p h} t\right)\right],
\end{array}
$$

where $n_{2}$ is the nonlinear refractive index.

\section{BASIC SYSTEM OF EQUATIONS}

The dynamics of narrow-band laser pulses can be accurately described in the frame of paraxial optics. The filamentation experiments demonstrate a typical pulse evolution: the initial laser pulse $\left(t_{0} \geq 50 \mathrm{fs}\right)$ possesses a relatively narrow-band spectrum $\left(\Delta k_{z} \ll k_{0}\right)$ and during the process the initial self-focusing and self-compression broadens the spectrum significantly. The broad-band spectrum $\left(\Delta k_{z} \sim k_{0}\right)$ is one of the basic characteristics of the stable filament. The evolution of the filament can not be further described in the frame of the nonlinear paraxial optics because the paraxial optics works correctly for narrow-band laser pulses only.

Recently, the evolution of broad-band laser pulses was described correctly within different non-paraxial models such as UPPE [15] or non-paraxial envelope equations [18]. Another standard restriction in the filamentation theory is the use of one-component scalar approximation of the electrical field $\vec{E}$. This approximation, though, is in contradiction with recent experimental results, where rotation of the polarization vector is observed [7]. For this reason in the present paper we use the non-paraxial vector model up to second order of dispersion, in which the nonlinear effects are described by the nonlinear polarization components (8). The system non-paraxial equations of the amplitude functions $A_{+}$and $A_{-}$has the form

$$
\begin{aligned}
-2 i k_{0}\left(\frac{\partial A_{+}}{\partial z}+\frac{1}{v_{g r}} \frac{\partial A_{+}}{\partial t}\right)=\Delta A_{+} & -\frac{1+\beta}{v_{g r}^{2}} \frac{\partial^{2} A_{+}}{\partial t^{2}} \\
& +k_{0}^{2} \tilde{n}_{2} A_{+}^{2} A_{-} \\
2 i k_{0}\left(\frac{\partial A_{-}}{\partial z}+\frac{1}{v_{g r}} \frac{\partial A_{-}}{\partial t}\right)=\Delta A_{-} & -\frac{1+\beta}{v_{g r}^{2}} \frac{\partial^{2} A_{-}}{\partial t^{2}} \\
& +k_{0}^{2} \tilde{n}_{2} A_{-}^{2} A_{+}
\end{aligned}
$$

where $\Delta$ is $3 D-(x, y, z)$ Laplace operator, $v_{g r}$ is the group velocity, $\beta=k_{0} v_{g r}^{2} k^{\prime \prime}$ and $k^{\prime \prime}$ is the group velocity dispersion. In the process of deriving of the system of Eqs. (9) is used in mind, that the left-hand and righthand components are complex conjugated fields.

This model describes the ionization-free filamentation regime and we will investigate the case, when the pulse intensities are slightly above to the critical one for selffocusing. The dispersion number in air is too small $\beta=k_{0} v_{g r}^{2} k^{\prime \prime} \simeq 2.1 \times 10^{-5}$ and we will use Eqs. (9) in approximation of the first order of dispersion.

$$
\begin{aligned}
& -2 i k_{0}\left(\frac{\partial A_{+}}{\partial z}+\frac{1}{v_{g r}} \frac{\partial A_{+}}{\partial t}\right)=\Delta A_{+}-\frac{1}{v_{g r}^{2}} \frac{\partial^{2} A_{+}}{\partial t^{2}} \\
& +\gamma A_{+}^{2} A_{-} \\
& 2 i k_{0}\left(\frac{\partial A_{-}}{\partial z}+\frac{1}{v_{g r}} \frac{\partial A_{-}}{\partial t}\right)=\Delta A_{-}-\frac{1}{v_{g r}^{2}} \frac{\partial^{2} A_{-}}{\partial t^{2}} \\
& { }_{+} \gamma A_{-}^{2} A_{+} \text {, }
\end{aligned}
$$

where $\gamma=n_{2} k_{0}^{2} A_{0}^{2}$ is nonlinear coefficient.

\section{VECTOR SOLITON SOLUTION AND VECTOR ROTATION. THE FILAMENT AS ROGUE WAVE}

The nonlinear system of equation (10) admits exact solitary vector solution, when $\gamma=2$ and the spectral width of the pulses reach the value $\Delta k_{z} \approx k_{0}$

$$
\begin{aligned}
& A_{+}(x, y, z, t)=\frac{2}{1+\tilde{r}^{2}} \exp \left[-i \Delta k_{z}\left(z-v_{g r} t\right)\right] \\
& A_{-}(x, y, z, t)=\frac{2}{1+\tilde{r}^{2}} \exp \left[i \Delta k_{z}\left(z-v_{g r} t\right)\right]
\end{aligned}
$$

where $\tilde{r}=\sqrt{x^{2}+y^{2}+\left(z-i k_{c e p}\right)^{2}-v_{g r}^{2}\left(t-i k_{c e p} / v_{g r}\right)^{2}}$ and $k_{c e p}$ is determinate below. The solution of the corresponding vector electrical field can be written after multiplying the amplitude functions (11) with the main phase (3)

$$
\begin{gathered}
E_{+}(x, y, z, t)=\frac{2}{1+\tilde{r}^{2}} \exp \left[-i \Delta k_{z}\left(v_{p h}-v_{g r}\right) t\right] \\
E_{-}(x, y, z, t)=\frac{2}{1+\tilde{r}^{2}} \exp \left[i \Delta k_{z}\left(v_{p h}-v_{g r}\right) t\right] .
\end{gathered}
$$

Lets turn from the left-hand and right-hand circular components (7]) to the standard Cartesian coordinates

$$
E_{x}=\left(E_{+}+E_{-}\right) / \sqrt{2}, E_{y}=\left(E_{+}-E_{-}\right) /(i \sqrt{2}) .
$$

The solution (12) written in Cartesian coordinates has the form

$$
\begin{aligned}
& E_{x}(x, y, z, t)=\frac{2}{1+\tilde{r}^{2}} \cos \left[\Delta k_{z}\left(v_{p h}-v_{g r}\right) t\right] \\
& E_{y}(x, y, z, t)=\frac{2}{1+\tilde{r}^{2}} \sin \left[\Delta k_{z}\left(v_{p h}-v_{g r}\right) t\right] .
\end{aligned}
$$


a)

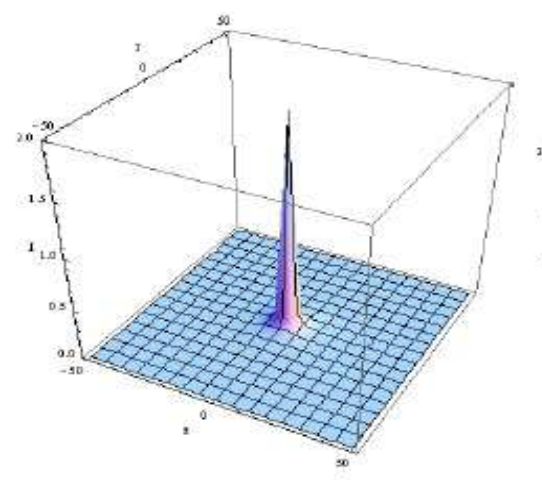

$\mathbf{z}=\mathbf{0}$ b)

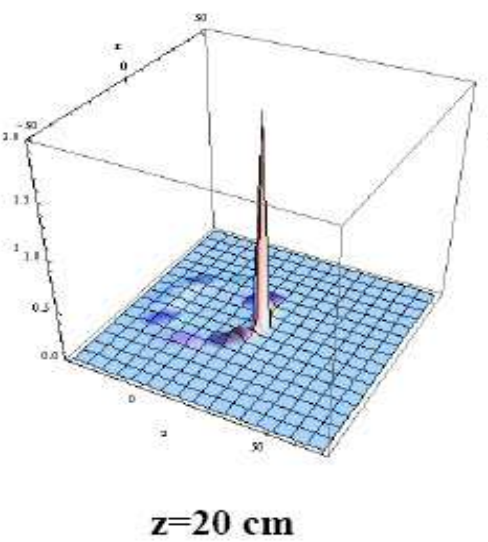

c)

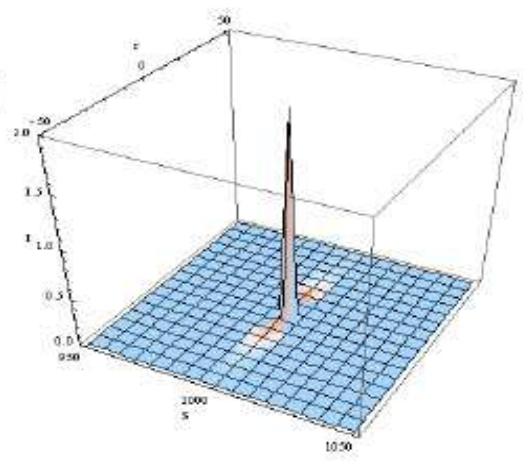

$\mathbf{z}=10 \mathrm{~m}$

FIG. 1. Evolution of the intensity profile $I=\left|A_{x}\right|^{2}+\left|A_{y}\right|^{2}$ of the solution (14). Side $(x, z)$ projection is plotted. On Fig.1a) is the initial profile, while on Fig.1b) the evolution of the intensity profile on distance $\mathrm{z}=20 \mathrm{~cm}$ is presented. It is clearly seen the generation of weak spherical wave. At large distances the solution is relatively stable, as it can be seen from the Fig.1c). One more detailed picture of the propagation in the far-away zone demonstrate a weak self-compression of the pulse.

The $3 D+1$ Lorentz type solution (14), presented in Cartesian coordinates, gives oscillation of the electrical vector $\vec{E}=\left(E_{x}, E_{y}, 0\right)$ in $(x, y)$ plane. It can be seen directly that the frequency of oscillation is equal to the carrier to envelope ones $\omega_{c e p}=k_{0}\left(v_{p h}-v_{g r}\right)$. The corresponding longitudinal spatial carrier to envelope wave number is $k_{c e p}=\omega_{c e p} / v_{g r}$. In standard atmosphere this complies to a rotation of the electrical field vector with period in $z$ direction $\lambda_{\text {cep }} \cong 10-20 \mathrm{~cm}$. Other important characteristic of the vector solution (14) is that it is soliton in $3+1$ dimensional complex Minkowski space and his real dynamics in 3D space is more close to weak Rogue wave with small self-compressing in the direction of propagation. The evolution of the intensity profile is plotted on Fig.1 and demonstrate a generation of week spherical wave (Fig 1. b) in the first milliseconds of the propagation distance. At large distances, as it can be seen from the Fig. 1c), the propagation is relatively stable with preserving the amplitude maximum. One more detailed picture of the propagation in the far-away zone demonstrate a weak self-compression of the pulse.

\section{CONCLUSIONS}

In this investigation we point that in the femtosecond region it is impossible to reduce the nonlinearity of third order to the scalar Kerr type only (proportional to the intensity). Therefore, we study more precisely the vector character of the nonlinear third order polarization, taking into account the carrier to envelope phase. The corresponding amplitude equations are written in terms of left-hand and right-hand circularly polarizing compo- nents. The vector system (10) admits exact $(3+1) \mathrm{D}$ soliton solutions (11) of Lorentz type. Our soliton solution is obtained for broad-band pulses $\Delta k_{z} \approx k_{0}$ and leads to the conclusion that the soliton appears as a balance between parabolic divergent type wave diffraction and parabolic convergent nonlinearity. The solution gives also a rotation of the vector of the electrical field with period equal to the period of carrier to envelope wavelength $\lambda_{c e p} \cong 10-20 \mathrm{~cm}$, in depends from the air pressure and temperature. Other important result is the generation of weak spherical wave in the first milliseconds of propagation and weak self compression in the far-away zone.

* lubomirkovach@yahoo.com

[1] A. Braun, G. Korn, X. Liu, D. Du, J. Squier, and G. Mourou, Opt. Lett. 20, 73-75 (1995).

[2] L. Wöste, C. Wedekind, H. Wille, P. Rairoux, B. Stein, S. Nikolov, C. Werner, S.Nierdermeier, F. Ronneberger, H. Schillinger, and R. Sauerbrey, AT-Fachverlag, Stuttgard, Laser and Optoelectronik 29, 51-53 (1997).

[3] S. Tzortzakis, G. Méchain, G. Patalano, Y.-B. André, B. Prade, M. Franco, A. Mysyrowicz, J. M. Munier, M. Gheudin, G. Beaudin, and P. Encrenaz, Opt. Lett. 27, 1944-1946, (2002).

[4] C. D'Amico, A. Houard, M. Franco, B. Prade, A. Mysyrowicz, Optics Express 15, 15274-15279 (2007).

[5] C. P. Hauri, A. Guandalini, P. Eckle, W. Kornelis, J. Biegert, U. Keller, Optics Express 13, 7541 (2005).

[6] S. L. Chin, A. Brodeur, S. Petit, O. G. Kosareva, V. P. Kandidov, J. Nonlinear Opt. Phys. Mater. 8, 121-146 (1999).

[7] O.Kosareva et al., Opt. Lett. 35, 2904-2906 (2010). 
[8] I. G. Koprinkov, A. Suda, P. Wang and K. Midorikawa, Phys. Rev. Lett. 84, 3847 (2000).

[9] S. L. Chin, S. A. Hosseini, W. Liu, Q. Luo, F. Théberge, N. Aközbek, A. Becker, V. P. Kandidov, O. G. Kosareva, and H. Schoeder, Can. J. Phys. 83, 863-905 (2005).

[10] P. Béjot, J. Kasparian, S. Henin, V. Loriot, T. Viellard, E. Hertz, O. Faucher, B. Lavorel, and J.-P. Wolf, Phys. Rev. Lett., 104, 103903 (2010).

[11] G. Méchain, A. Couairon, Y.-B. André, C. D'Amico, M. Franco, B. Prade, S. Tzortzakis, A. Mysyrowicz, R. Sauerbrey, Appl.Phys. B 79, 379-382 (2004).

[12] G. Méchain, Study of filamentation of femtosecond laser pulses in air, These de doctorat, Ecole Polytechnique, Palaiseau, France, 2005.

[13] Magali Durand et al., Optics Express, 21, 26836 (2013).
[14] P. D. Maker and R. W. Terhune, Phys. Rev. 137, A801 (1965).

[15] M. Kolesik, J. V. Moloney, Optics Express, 16, 2971 (2008).

[16] M. Kolesik, E. M. Wright, A. Becker, J. V. Moloney, Appl. Phys. B, 85, pp 531-538 (2006).

[17] L. M. Kovachev, Journal of Modern Optics, 56, 17971803 (2009).

[18] Lubomir M. Kovachev and Kamen Kovachev, Laser System for Applications, Chapter 11, InTech, 2011.

[19] R. W. Boyd Nonlinear Optics, ( 3th ed., Academic Press, 2003).

[20] G. P. Agrawal, Nonlinear Fiber Optics, (4th ed. Academic, 2007). 